Steinemann, A., Nematollahi, N., Weinberg, J.L., Flattery, J., Goodman, N. and Kolev, S.D. (2020) Volatile chemical emissions from car air fresheners. Air Quality, Atmosphere \& Health, pp.1-6.DOI: https://doi.org/10.1007/s11869-020-00886-8

\title{
Volatile Chemical Emissions from Car Air Fresheners
}

Anne Steinemann*, Neda Nematollahi, Justine Lew Weinberg, Jennifer Flattery, Nigel Goodman, Spas D. Kolev

\section{Anne Steinemann*}

Professor of Civil Engineering, Department of Infrastructure Engineering, Melbourne School of Engineering, The University of Melbourne, Parkville Victoria 3010 Australia

Professor of Engineering, Chair of Sustainable Infrastructure, College of Science and Engineering, James Cook University, Townsville Queensland 4814 Australia

*Corresponding author. Email address: anne.steinemann@unimelb.edu.au

\section{Neda Nematollahi}

Postdoctoral Research Fellow, Department of Infrastructure Engineering, Melbourne School of Engineering, The University of Melbourne, Parkville Victoria 3010 Australia

\section{Justine Lew Weinberg}

MSEHS, CIH, Public Health Institute, contractor to Occupational Health Branch, California Department of Public Health, Richmond California 94804 United States

\section{Jennifer Flattery}

M.P.H., Research Scientist Supervisor, Occupational Health Branch, California Department of Public Health, Richmond California 94804 United States

\section{Nigel Goodman}

Vice Chancellor's Postdoctoral Research Fellow, School of Property Construction and Project Management, RMIT University, Melbourne VIC 3000 Australia

\section{Spas D. Kolev}

Professor of Chemistry, School of Chemistry, The University of Melbourne, Parkville Victoria 3010 Australia

Keywords: car air freshener, fragrance, volatile organic compounds, emissions

This is a pre-print of an article published in Air Quality, Atmosphere \& Health. The final authenticated version is available online at: https://doi.org/10.1007/s11869-020-00886-8 


\title{
Volatile Chemical Emissions from Car Air Fresheners
}

\begin{abstract}
Air fresheners, used in a variety of indoor environments, emit a range of volatile chemicals, including some classified as hazardous. However, little is known about the emissions from air fresheners designed for use in cars. This study investigates the volatile organic compounds emitted from car air fresheners, identifies potentially hazardous compounds, compares emissions between so-called natural and regular versions, and assesses whether ingredients are disclosed. Using gas chromatography/mass spectrometry, 12 car air fresheners were analysed for their volatile emissions. Air freshener types included car vent clips, wraps, hanging ornaments, cans, and spray. Results reveal that the air fresheners collectively emitted 546 VOCs with 30 VOCs classified as potentially hazardous. All air freshener types emitted one or more potentially hazardous compounds. Comparing regular air fresheners with socalled natural or green air fresheners, no significant difference was found in the emissions of hazardous compounds. Notably, all products emitted at least one VOC classified as potentially hazardous. Among all of the 546 compounds emitted, fewer than $2 \%$ of all VOCs, and none of the potentially hazardous VOCs, were disclosed on any product label or safety data sheet. This study reveals that car air fresheners can be a source of exposure to numerous volatile compounds, including potentially hazardous VOCs, most of which are undisclosed. Of particular concern for human exposure is the small and enclosed breathing space within vehicles, as well as involuntary exposure in commercial vehicles such as taxi cabs and rideshares.
\end{abstract}




\section{Introduction}

Air fresheners are pervasive within indoor environments, from public and private buildings to forms of transportation. Although air fresheners may be used with the intent to enhance environments, studies reveal that these products generally impair rather than improve indoor air quality.

Chemical analyses of air fresheners have found they emit a range of volatile organic compounds (VOCs), such as terpenes (e.g., limonene, linalool, alpha-pinene, beta-pinene), including some VOCs classified as hazardous, such as acetaldehyde (Steinemann 2017). In addition, primary emissions of common air freshener chemicals (terpenes) can readily react with ozone to generate additional and increased concentrations of hazardous pollutants, such as formaldehyde (Nazaroff and Weschler 2004).

Although prior research has investigated emissions from air fresheners used primarily in buildings (e.g., Steinemann 2017, Goodman et al. 2019, Uhde and Shulz 2015, Nematollahi et al. 2019), little prior work has analysed emissions from air fresheners used in cars. These types of air fresheners include car vent wraps and clips, hanging ornaments such as trees, cans, and sprays, which can comprise aromatic solids, liquids, gels, or oils. Sources of exposure include not only privately owned vehicles, but also publicly available vehicles such as rental cars, rideshares, and taxi cabs. Within the small and enclosed space in cars, exposures may be of particular concern, both for the driver and passengers.

Air freshener exposures have been associated with a range of adverse health effects, including asthma symptoms, breathing difficulties, migraine headaches, and dizziness (Weinberg et al. 2017, Steinemann 2019a, 2019b, 2018, 2017, Steinemann and Nematollahi 2020, Steinemann and Goodman 2019). Reducing risks from air freshener exposures is vital not only for occupant health but also for public safety. For instance, car drivers and passengers may experience acute effects while in the car, as well as longer term effects after leaving the car. Further, air freshener chemicals can continue to be emitted within interior spaces, such as within cars, even after the air fresheners are removed (Goodman et al. 2019, Yoshida et al. 2006). 
The purpose of this study was to investigate emissions from a range of common air fresheners used in cars. It pursues four primary objectives: (a) to analyse the volatile ingredients emitted from car air fresheners, (b) to identify the compounds classified as potentially hazardous, (c) to compare emissions between so-called natural and regular air fresheners, and (d) to assess whether compounds emitted are disclosed on product labels or safety data sheets. Findings from this study offer a new body of data and increased understanding of our exposure to volatile compounds from car air fresheners, and point to the importance of reducing exposure to reduce potential health and safety risks.

\section{Methods}

For this study, 12 car air fresheners were a convenience sample randomly selected from the automotive supply or air freshener section of stores in California. However, the same brands and types of products are available across the United States and internationally. In this paper, the term "natural" refers to products that make the claim of being "natural" or related terms, such as "organic," "no chemicals," "good for the environment," or "healthier and cleaner fragrance." The term "regular" refers to products other than those in the "natural" category. The types of air fresheners sampled were 6 car vent air fresheners ( 2 natural, 4 regular), 3 hanging air fresheners ( 3 regular), 2 can air fresheners ( 2 natural), and 1 spray air freshener ( 1 regular). For each air freshener, the product was maintained in its original packaging until the time of analysis, and a sample of approximately $2 \mathrm{~g}$ was placed inside a $10 \mathrm{~mL}$ amber vial.

The volatile emissions were analysed by headspace gas chromatography/mass spectromety (GC/MS), using a Shimadzu GC/MS-QP2010 Plus instrument equipped with a BPX-VOL capillary column and coupled to an automated Shimadzu AOC-500 sample injection system. The chromatogram for each air freshener was scanned to identify the highest concentration VOCs and the potentially hazardous compounds. Compound identification was based on the mass spectral library of the National Institute of Standards and Technology NIST Version 2.0. For additional details on the GC/MS method and compound identification procedure, see Nematollahi et al. (2018).

Potentially hazardous VOCs were identified according to their classification as (i) Hazardous Air Pollutants, HAPs (EPA 2017) including carcinogenic HAPs (EPA 2018), (ii) California Proposition 65 chemicals (OEHHA 2020), or (iii) asthmagens (AOEC 2020). This analysis was performed to identify ingredients of the air fresheners studied that are classified as 
potentially hazardous under one or more of these laws or guidelines mentioned above. However, this analysis does not imply that the entire product is potentially hazardous. It also does not imply that the VOCs identified are the only potentially hazardous compounds contained in the air fresheners studied.

\section{Results}

VOCs emitted and most prevalent VOCs

A summary of VOCs emitted across the 12 air fresheners, and from the subsets of "regular" and "natural" car air fresheners, is provided in Table 1. In this paper, the term "VOC occurrences" refers to the number of individual VOCs emitted from each car air freshener. The term "VOC identities" refers to the number of uniquely named VOCs emitted from one or more of the car air fresheners. Thus, a VOC occurrence is an individual ingredient emitted from one product, and a VOC identity is a VOC that occurs in one or more of the products.

Across the 12 car air fresheners, 546 VOCs were emitted (occurrences), representing 275 unique VOCs (identities). The most prevalent VOCs (in at least $75 \%$ of all car air fresheners) were limonene, benzyl acetate, acetone, ethanol, linalool, 2-methylbutyl acetate, acetaldehyde, and methanol (Table 2 and Supplementary Table 2). In "regular" car air fresheners, the most prevalent VOC was limonene. In "natural" car air fresheners, the most prevalent VOC was benzyl acetate.

\section{Potentially hazardous emissions}

To identify potentially hazardous emissions, three classifications (HAPs, Prop 65, asthmagens) were used to assess the emitted VOCs (see Tables 1 and 3).

For the 275 VOCs (identities) emitted across the 12 products, 9 VOCs are classified as potentially hazardous: methanol, acetaldehyde, beta-myrcene, 3-carene, o-xylene, hexane, tetracarbonylnickel, toluene, and propanal.

For the 546 VOCs (occurrences) emitted collectively from the 12 products, 30 VOCs are classified as potentially hazardous, representing approximately $6 \%$ of all VOC ingredients. All products emitted at least one VOC classified as potentially hazardous. 
Comparison of VOCs emitted from regular and natural products

Among the most prevalent VOCs, no significant difference was found in VOC occurrences between the regular and natural products $(\mathrm{p}=0.12, \mathrm{t}$ test). In addition, among the potentially hazardous VOCs, no significant difference was found in VOC occurrences between regular and natural products $(\mathrm{p}=0.16, \mathrm{t}$ test). This comparison followed the convention of previously published work (e.g., Steinemann 2015, Nematollahi et al. 2019).

\section{Comparison of VOCs emitted and ingredients disclosed}

Among the 546 VOCs (occurrences) emitted from the products, 10 were listed on any product label or safety data sheet. In addition, none of the 30 VOCs classified as potentially hazardous were listed on any product label or safety data sheet. Thus, fewer than $2 \%$ of all VOCs, and none of the potentially hazardous VOCs, were disclosed to the public on product labels or safety data sheets.

\section{Discussion}

This study found that car air fresheners of all types, including both regular and natural versions, emit numerous volatile chemicals, some of which are classified as hazardous, and few of which are disclosed to the public. Car air freshener emissions are of particular concern, given that car interiors are small and enclosed spaces that can be subject to air pollutants from both outdoor and indoor sources, and thus air fresheners represent an additional source of pollutants and health risks. In addition, air fresheners, which add a chemical mixture into the air space, are designed to impart a scent or mask an odor, rather than to reduce air pollutants or health risks. Further, vent clips, because they attach directly to the car air vent, turn the ventilation system, which could be a source of fresher air, into a source for dispensing potentially hazardous VOCs into the enclosed space.

Our findings are consistent with prior studies of car air freshener emissions. Lamorena and Lee (2008), in their analysis of a car air freshener, found terpenes (alpha-pinene, beta-pinene, p-cymene, and limonene) as primary constituents, and their ozone-initiated reaction products (including formaldehyde, acetaldehyde, acrolein, acetone, and propionaldehyde), deemed to affect the health of passengers. Yoshida et al. (2006), in their analysis of 25 car air fresheners, 
found tepenes (limonene, beta-pinene, 1,8-cineole, linalool, and dihydromyrcenol) and esters (ethylacetate, ethylbutyrate, and isopentylacetate) as major volatile components. Importantly, even after air fresheners were removed from vehicles, their volatile components continued to adversely affect air quality.

Our results are also consistent with broader findings on air fresheners. First, in prior studies of air freshener emissions (e.g., Steinemann 2017, 2015, Nematollahi et al. 2019, Uhde and Schulz 2015, Kim et al. 2015), terpenes and esters were also among the most common compounds. Second, all air freshener types, including so-called organic and natural essential oils, emitted potentially hazardous VOCs (e.g., Steinemann 2017, 2015, Nematollahi et al. 2018, 2019). Third, in comparisons of natural and regular air fresheners, no significant difference was found in emissions of potentially hazardous pollutants (Steinemann 2015, Nematollahi 2019). Fourth, in comparisons of VOCs emitted and ingredients listed (e.g., Steinemann 2015, Nematollahi et al. 2019, Uhde and Schulz 2015), fewer than 10\% of volatile ingredients in air fresheners were disclosed.

To note, the GC/MS headspace analysis identified compounds that are individual ingredients in the product, without interactions with external constituents. With this focus on individual compounds and primary emissions, the analysis did not examine mixtures of compounds or the generation of secondary pollutants that could contribute to the overall risk. However, our results provide an important foundation for a more complete assessment to determine exposure situations and quantify associated risks.

Finally, as studies have revealed, people prefer fragrance-free rather than fragranced indoor environments. In nationally representative population-based surveys in five countries (the United States, Australia, United Kingdom, Sweden, and Germany), a majority of the general public prefers hotels, airplanes, and workplaces that are fragrance-free rather than fragranced, and will choose options without a scent rather than with a scent (Steinemann 2019a, 2019b, Steinemann and Klaschka 2019). An even greater percentage of vulnerable individuals, such as those with asthma, autism, or chemical sensitivity, prefer fragrance-free to fragranced indoor environments (Steinemann 2019a, 2019b, 2018). Thus, people may similarly prefer indoor car environments that are without a fragranced product rather than with a fragranced product such as an air freshener. Drivers of taxi cabs and rideshares may thus be mistakenly assuming that their passengers prefer the use of air fresheners. 


\section{Conclusions}

Our study found that car air fresheners emit numerous volatile chemicals, including potentially hazardous air pollutants. Given that air freshener emissions are associated with health risks, that air fresheners are not intended to reduce pollutants, that driver health is imperative to public safety, that passenger risks may often be involuntary, and that people generally prefer fragrance-free to fragranced indoor environments, it would seem reasonable to rethink the pervasive use of air fresheners within cars.

\section{Acknowledgments}

We thank the supporters of this study: the Clean Air and Urban Landscapes Hub funded by the Australian Government's National Environmental Science Program; and the Commonwealth Scientific and Industrial Research Organisation (CSIRO). We also thank the anonymous reviewers of this paper. 


\section{References}

(AOEC) Association of Occupational and Environmental Clinics. (2020) Exposure Code List. Asthmagens. http://www.aoecdata.org/ (accessed April 25, 2020)

(EPA) Environmental Protection Agency (2017) Initial List of Hazardous Air Pollutants with Modifications https://www.epa.gov/haps/initial-list-hazardous-air-pollutants-modifications (accessed April 25, 2020)

(EPA) Environmental Protection Agency (2018) Prioritized Chronic Dose-Response Values. Weight of evidence for carcinogenicity https://www.epa.gov/sites/production/files/201405/documents/table1.pdf (accessed April 25, 2020)

Goodman NB, Nematollahi N, Agosti G, Steinemann A. 2019. Evaluating air quality with and without air fresheners. Air Quality, Atmosphere \& Health. 13(1):1-4.

Kim S, Hong SH, Bong CK, Cho MH (2015) Characterization of air freshener emission: the potential health effects. The Journal of toxicological sciences 40(5):535-550.

Lamorena RB, Lee W (2008) Influence of ozone concentration and temperature on ultra-fine particle and gaseous volatile organic compound formations generated during the ozoneinitiated reactions with emitted terpenes from a car air freshener. Journal of hazardous materials. 158(2-3):471-7.

Nazaroff WW, Weschler CJ (2004) Cleaning products and air fresheners: exposure to primary and secondary air pollutants. Atmospheric environment 38(18):2841-2865.

Nematollahi N, Kolev SD, Steinemann A. 2018. Volatile chemical emissions from essential oils. Air Quality, Atmosphere \& Health. 11(8):949-54.

Nematollahi N, Kolev SD, Steinemann A. 2019. Volatile Chemical Emissions from 134 Common Consumer Products. Air Quality, Atmosphere \& Health. 12(11):1259-65.

(OEHHA) The Proposition 65 List (2020) Chemicals that are known to cause cancer or birth defects or other reproductive harm https://oehha.ca.gov/proposition-65/proposition-65-list

Steinemann A. 2015. Volatile Emissions from Common Consumer Products. Air Quality, Atmosphere and Health 8(3):273-281.

Steinemann A. 2017. Ten Questions Concerning Air Fresheners and Indoor Built Environments. Building and Environment 111:279-284.

Steinemann A. 2018. Fragranced Consumer Products: Effects on Autistic Adults in the United States, Australia, and United Kingdom. Air Quality, Atmosphere and Health 11(10):1137-1142.

Steinemann A. 2019a. International Prevalence of Fragrance Sensitivity. Air Quality, Atmosphere and Health 12(8):891-897.

Steinemann A. 2019b. Ten Questions concerning Fragrance-Free Policies and Indoor Environments. Building and Environment 159:1-8. 
Steinemann A, Goodman N. 2019. Fragranced Consumer Products and Effects on Asthmatics: An International Population-based Study. Air Quality, Atmosphere and Health 12(6):643-649.

Steinemann A, Klaschka U. 2019. Exposures and Effects from Fragranced Consumer Products in Germany 12:1399-1404.

Steinemann A, Nematollahi N. 2020. Migraine headaches and fragranced consumer products: an international population-based study. Air Quality, Atmosphere \& Health. 1-4.

Uhde E, Schulz N (2015) Impact of room fragrance products on indoor air quality. Atmospheric Environment 106:492-502.

Weinberg JL, Flattery J, Harrison R (2017) Fragrances and work-related asthma-California surveillance data, 1993-2012. J Asthma 54:1041-1050.

Yoshida T, Matsunaga I, Tomioka K, Kumagai S (2006) Interior air pollution in automotive cabins by volatile organic compounds diffusing from interior materials: I. Survey of 101 types of Japanese domestically produced cars for private use. Indoor and Built Environment. $15(5): 425-44$. 
Table 1: VOCs emitted from the car air fresheners studied*

Emitted

\begin{tabular}{ll} 
Type & \\
\hline Regular & 8
\end{tabular}

Natural

4

Total
Number of car air fresheners

8

12

All emitted
VOCs
389 occurrences
232 identities

Potentially Hazardous VOCs

23 occurrences

8 identities

157 occurrences

114 identities

7 occurrences
4 identities

546 occurrences

275 identities

\section{0 occurrences} 9 identities
Listed

(on product label or safety data sheet)

All listed VOCs Hazardous VOCs

5 occurrences $\quad 0$ occurrences

5 identities $\quad 0$ identities

5 occurrences $\quad 0$ occurrences

5 identities

0 identities

10 occurrences 10 identities

*"VOC occurrences" refers to the number of individual VOCs emitted from each car air freshener.

"VOC identities" refers to the number of unique VOCs emitted from one or more of the car air fresheners. 
Table 2: Most prevalent VOCs emitted from the car air fresheners studied

\begin{tabular}{|c|c|c|c|c|}
\hline \multirow[t]{2}{*}{ Compound } & \multirow[t]{2}{*}{ CAS \# } & \multicolumn{3}{|c|}{ Prevalence (\# of car air fresheners) } \\
\hline & & $\begin{array}{l}\text { Total } \\
(\mathrm{n}=12)\end{array}$ & $\begin{array}{l}\text { Regular } \\
(\mathrm{n}=8)\end{array}$ & $\begin{array}{l}\text { Natural } \\
(\mathrm{n}=4)\end{array}$ \\
\hline \multicolumn{5}{|l|}{ All car air fresheners $(n=12)$} \\
\hline Limonene & $138-86-3$ & 10 & 8 & 2 \\
\hline Benzyl acetate & $140-11-4$ & 9 & 5 & 4 \\
\hline Acetone & $67-64-1$ & 9 & 7 & 2 \\
\hline Ethanol & $64-17-5$ & 9 & 6 & 3 \\
\hline Linalool & $78-70-6$ & 8 & 6 & 2 \\
\hline 2-Methylbutyl acetate & $624-41-9$ & 8 & 4 & 4 \\
\hline Acetaldehyde* & $75-07-0$ & 8 & 7 & 1 \\
\hline Methanol* & $67-56-1$ & 8 & 5 & 3 \\
\hline $\begin{array}{l}\text { 3-Penten-2-one, } 4-(2,6,6- \\
\text { trimethyl-2-cyclohexen-1-yl) }\end{array}$ & $114933-28-7$ & 7 & 4 & 3 \\
\hline beta-Myrcene* & $123-35-3$ & 7 & 5 & 2 \\
\hline Dihydromyrcenol & $18479-58-8$ & 7 & 5 & 2 \\
\hline Isoamyl acetate & $123-92-2$ & 7 & 4 & 3 \\
\hline Neryl acetate & $141-12-8$ & 7 & 6 & 1 \\
\hline 4-tert-Butylcyclohexyl acetate & $32210-23-4$ & 6 & 4 & 2 \\
\hline Eucalyptol & $470-82-6$ & 6 & 4 & 2 \\
\hline Sabinene & $3387-41-5$ & 6 & 4 & 2 \\
\hline Verdyl acetate & $5413-60-5$ & 6 & 5 & 1 \\
\hline
\end{tabular}

$\underline{\text { Regular car air fresheners }(\mathrm{n}=8)}$

Limonene

Acetone

Acetaldehyde*

Ethanol

Linalool

Neryl acetate

Benzyl acetate

Methanol*

beta-Myrcene*

Dihydromyrcenol

Verdyl acetate

alpha-Terpineol
138-86-3 8

67-64-1 7

$75-07-0 \quad 7$

$64-17-5 \quad 6$

$78-70-6 \quad 6$

141-12-8 6

140-11-4 5

$67-56-1 \quad 5$

123-35-3 5

$18479-58-8 \quad 5$

5413-60-5 5

$98-55-5 \quad 5$

Natural car air fresheners $(\mathrm{n}=4)$

Benzyl acetate 
2-Methylbutyl acetate

624-41-9

4

Ethanol

64-17-5

3

Methanol*

67-56-1

3

3-Penten-2-one, 4-(2,6,6-

114933-28-7 3

trimethyl-2-cyclohexen-1-yl)

Isoamyl acetate

123-92-2 3

Ethyl butyrate

$105-54-4$

3

Benzaldehyde

$100-52-7$

3

4,4'-Isopropylidenebis(2,6-

$5613-46-7$

3

dimethylphenol)

*Classified as potentially hazardous 
Table 3: Potentially hazardous VOCs emitted from the car air fresheners studied

\begin{tabular}{llllll}
\hline Compound & CAS \# & $\begin{array}{l}\text { Prevalence } \\
\text { (\# of Products) }\end{array}$ & HAPs & Prop65 & Asthmagens \\
& & & & \\
\hline Methanol & $67-56-1$ & 8 & $\checkmark$ & $\checkmark$ & \\
Acetaldehyde $^{c}$ & $75-07-0$ & 8 & $\checkmark$ & $\checkmark$ & \\
beta-Myrcene & $123-35-3$ & 7 & & $\checkmark$ & \\
3-Carene & $13466-78-9$ & 2 & & & $\checkmark$ \\
o-Xylene & $95-47-6$ & 1 & $\checkmark$ & & \\
Hexane & $110-54-3$ & 1 & $\checkmark$ & $\checkmark$ & \\
Tetracarbonylnickel & $13463-39-3$ & 1 & & $\checkmark$ & $\checkmark$ \\
Toluene & $108-88-3$ & 1 & $\checkmark$ & $\checkmark$ & \\
Propanal & $123-38-6$ & 1 & $\checkmark$ & & \\
\hline
\end{tabular}

HAPs: United States Environmental Protection Agency (EPA) - Hazardous Air Pollutants (EPA 2017)

Prop65: California Proposition 65 (OEHHA 2020)

Asthmagens: Association of Occupational and Environmental Clinics (AOEC 2020)

${ }^{\mathrm{c}}$ Classified as possibly carcinogenic (2B) (EPA 2018) 


\section{Supplementary Table 1: VOCs emitted from each of the car air fresheners studied}

\section{Car spray air freshener}

\begin{tabular}{ll}
\hline Compounds & CAS\# \\
\hline Isopropyl alcohol & $67-63-0$ \\
\hline Butane & $106-97-8$ \\
\hline Limonene & $138-86-3$ \\
\hline Isoamyl acetate & $123-92-2$ \\
\hline Eucalyptol & $470-82-6$ \\
\hline D,L-isobornyl acetate & $92618-89-8$ \\
\hline Isopentane & $78-78-4$ \\
\hline alpha-Pinene & $80-56-8$ \\
\hline Acetone & $67-64-1$ \\
\hline Pentane & $109-66-0$ \\
\hline 2-Methylbutyl acetate & $624-41-9$ \\
\hline Ethanol & $64-17-5$ \\
\hline Camphene & $79-92-5$ \\
\hline beta-Pinene & $127-91-3$ \\
\hline Acetaldehyde* & $75-07-0$ \\
\hline Propylene glycol & $57-55-6$ \\
\hline Methyl salicylate & $119-36-8$ \\
\hline beta-Myrcene* & $123-35-3$ \\
\hline beta-Citral & $106-26-3$ \\
\hline Terpinolene & $586-62-9$ \\
\hline Diisopropyl ether & $108-20-3$ \\
\hline Isopropyl formate & $625-55-8$ \\
\hline (E)-citral & $141-27-5$ \\
\hline N-butylbenzenesulfonamide & $3622-84-2$ \\
\hline Benzyl acetate & $140-11-4$ \\
\hline gamma-Terpinene & $99-85-4$ \\
\hline Sabinene & $3387-41-5$ \\
\hline Cyclofenchene & $488-97-1$ \\
\hline 1-Propanol & $71-23-8$ \\
\hline Hexane* & $110-54-3$ \\
\hline 2-Methylpentane & $107-83-5$ \\
\hline gamma-Terpineol & $586-81-2$ \\
\hline Triethylene glycol & $112-27-6$ \\
\hline beta-Phellandrene & $555-10-2$ \\
\hline o-Cymene & $527-84-4$ \\
\hline Compound coss \\
\hline
\end{tabular}

*Compound classified as potentially hazardous 


\section{Car can air freshener ${ }^{N}$}

\begin{tabular}{ll}
\hline Compounds & CAS\# \\
\hline Benzaldehyde & $100-52-7$ \\
\hline Ethyl butyrate & $105-54-4$ \\
\hline Isoamyl acetate & $123-92-2$ \\
\hline Benzyl acetate & $140-11-4$ \\
\hline o-Tolualdehyde & $529-20-4$ \\
\hline 2-Methylbutyl acetate & $624-41-9$ \\
\hline Amyl acetate & $628-63-7$ \\
\hline 4-tert-Butylcyclohexyl acetate & $32210-23-4$ \\
\hline Ethanol & $64-17-5$ \\
\hline p-tert-Butyl cyclohexyl-acetate cis & $10411-92-4$ \\
\hline Dimethyl succinate & $106-65-0$ \\
\hline 3-Penten-2-one, 4-(2,6,6-trimethyl-2-cyclohexen- & $114933-28-7$ \\
1-yl) & $134-20-3$ \\
\hline Methyl anthranilate & $67-56-1$ \\
\hline Methanol* & $19464-92-7$ \\
\hline trans-Ethyl 3-Methyl-3-phenylglycidate & $43047-93-4$ \\
\hline 10-(oxan-2-yloxy)decan-1-ol & $19464-95-0$ \\
\hline cis-Ethyl 3-methyl-3-phenylglycidate & $67-64-1$ \\
\hline Acetone & $97-62-1$ \\
\hline Ethyl isobutyrate & $104-87-0$ \\
\hline p-Tolualdehyde & $100-52-7$ \\
\hline Benzaldehyde & $105-54-4$ \\
\hline Ethyl butyrate & $123-92-2$ \\
\hline Isoamyl acetate & $140-11-4$ \\
\hline Benzyl acetate & $529-20-4$ \\
\hline o-Tolualdehyde &
\end{tabular}

*Compound classified as potentially hazardous

${ }^{N}$ Product with the claim of being "natural" or a related term. 


\section{Car can air freshener ${ }^{N}$}

\begin{tabular}{ll}
\hline Compounds & CAS\# \\
\hline Benzaldehyde & $100-52-7$ \\
\hline Ethyl butyrate & $105-54-4$ \\
\hline Amyl acetate & $628-63-7$ \\
\hline Benzyl acetate & $140-11-4$ \\
\hline o-Tolualdehyde & $529-20-4$ \\
\hline 2-Methylbutyl acetate & $624-41-9$ \\
\hline 3-Penten-2-one, 4-(2,6,6-trimethyl-2-cyclohexen- & $114933-28-7$ \\
1-yl) & \\
\hline Ethanol & $64-17-5$ \\
\hline N-butylbenzenesulfonamide & $3622-84-2$ \\
\hline trans-Ethyl 3-Methyl-3-phenylglycidate & $19464-92-7$ \\
\hline Methanol* & $67-56-1$ \\
\hline 4,4'-Isopropylidenebis(2,6-dimethylphenol) & $5613-46-7$ \\
\hline Methyl anthranilate & $134-20-3$ \\
\hline cis-Ethyl 3-methyl-3-phenylglycidate & $19464-95-0$ \\
\hline o-Xylene* & $95-47-6$ \\
\hline p-Tolualdehyde & $104-87-0$ \\
\hline 1-Methyl-alpha-ionone & $7779-30-8$ \\
\hline 1-Pentanol & $71-41-0$ \\
\hline$*$ Compound classified as potentially hazardous \\
$N$ Product with the claim of being "natural" or a related term.
\end{tabular}




\section{Car hanging air freshener}

\begin{tabular}{|c|c|}
\hline Compounds & CAS\# \\
\hline Allyl hexanoate & $123-68-2$ \\
\hline Limonene & $138-86-3$ \\
\hline Isoamyl butylate & $106-27-4$ \\
\hline Allyl heptanoate & $142-19-8$ \\
\hline Triacetin & $102-76-1$ \\
\hline gamma-Nonanolactone & $104-61-0$ \\
\hline Isoamyl acetate & $123-92-2$ \\
\hline Dipentyl ether & $693-65-2$ \\
\hline Verdyl acetate & $5413-60-5$ \\
\hline Ethyl lactate & $97-64-3$ \\
\hline$\gamma$-Undecalactone & $104-67-6$ \\
\hline 2-Methylbutyl acetate & $624-41-9$ \\
\hline o-Cymene & $527-84-4$ \\
\hline Allyl cyclohexanepropionate & $2705-87-5$ \\
\hline p-Anisaldehyde & $123-11-5$ \\
\hline Ethyl hexanoate & $123-66-0$ \\
\hline 4,4'-Isopropylidenebis(2,6-dimethylphenol) & $5613-46-7$ \\
\hline beta-Pinene & $127-91-3$ \\
\hline$\gamma$-Decalactone & $706-14-9$ \\
\hline 2,6-Di-tert-butyl-4-methylphenol & $128-37-0$ \\
\hline Diethyl malonate & $105-53-3$ \\
\hline Neryl acetate & $141-12-8$ \\
\hline Isopropyl alcohol & $67-63-0$ \\
\hline$\alpha, \alpha$-Dimethylphenethyl butyrate & $10094-34-5$ \\
\hline Allyl alcohol & $107-18-6$ \\
\hline beta-Myrcene* & $123-35-3$ \\
\hline Ethyl butyrate & $105-54-4$ \\
\hline Methanol* & $67-56-1$ \\
\hline
\end{tabular}

${ }^{*}$ Compound classified as potentially hazardous 
5. Car hanging air freshener

\begin{tabular}{|c|c|}
\hline Compounds & CAS\# \\
\hline Linalyl acetate & $115-95-7$ \\
\hline Linalool & $78-70-6$ \\
\hline Dihydromyrcenol & $18479-58-8$ \\
\hline Verdyl acetate & $5413-60-5$ \\
\hline Benzyl acetate & $140-11-4$ \\
\hline Isobornyl acetate & $125-12-2$ \\
\hline 4-tert-Butylcyclohexyl acetate & $32210-23-4$ \\
\hline Nopyl acetate & $128-51-8$ \\
\hline p-tert-Butyl cyclohexyl-acetate cis & $10411-92-4$ \\
\hline Eucalyptol & $470-82-6$ \\
\hline 2,9-Dimethylundecane & $17301-26-7$ \\
\hline 3-Methyl-5-propylnonane & $31081-18-2$ \\
\hline Limonene & $138-86-3$ \\
\hline 2,2,11,11-Tetramethyldodecane & $127204-12-0$ \\
\hline 2,2-Dimethyltetradecane & $59222-86-5$ \\
\hline Ethyl acetate & $141-78-6$ \\
\hline 6-Methyltridecane & $13287-21-3$ \\
\hline 3,8-Dimethyldecane & $17312-55-9$ \\
\hline 2-Bromotetradecane & $74036-95-6$ \\
\hline Terpinyl acetate & $80-26-2$ \\
\hline 4,6-Dimethyldodecane & $61141-72-8$ \\
\hline 2,6,6-Trimethyloctane & $54166-32-4$ \\
\hline 2,2,4-Trimethylhexane & $16747-26-5$ \\
\hline 2,5-Dimethylundecane & $17301-22-3$ \\
\hline 2,2,6-Trimethyldecane & $62237-97-2$ \\
\hline 3-Methylundecane & $1002-43-3$ \\
\hline 4-Terpineol & $562-74-3$ \\
\hline Acetone & $67-64-1$ \\
\hline 2,3,6-Trimethyldecane & $62238-12-4$ \\
\hline alpha-Pinene & $80-56-8$ \\
\hline alpha-Terpineol & $98-55-5$ \\
\hline Phytane & $638-36-8$ \\
\hline Methyl phenylcarbinyl acetate & $93-92-5$ \\
\hline Heneicosane & $629-94-7$ \\
\hline 2,2,7,7-Tetramethyloctane & $1071-31-4$ \\
\hline 3,3-Dimethylundecane & $17312-65-1$ \\
\hline 4,4-Dimethylundecane & $17312-68-4$ \\
\hline beta-Pinene & $127-91-3$ \\
\hline 2,5-Dimethyltridecane & $56292-66-1$ \\
\hline Heptadecane & $629-78-7$ \\
\hline Ethanol & $64-17-5$ \\
\hline Methanol* & $67-56-1$ \\
\hline Dicyclopentenyl alcohol & $37275-49-3$ \\
\hline 2,2,9-Trimethyldecane & $62238-00-0$ \\
\hline 10-Methylnonadecane & $56862-62-5$ \\
\hline 2,4-Dimethyl-3-pentanol & $600-36-2$ \\
\hline beta-Myrcene* & $123-35-3$ \\
\hline
\end{tabular}




\begin{tabular}{ll}
\hline Camphene & $79-92-5$ \\
\hline 1-(2-Methoxypropoxy)propan-2-ol & $13429-07-7$ \\
\hline 2,2,3-Trimethylnonane & $55499-04-2$ \\
\hline Neryl acetate & $141-12-8$ \\
\hline beta-Terpinyl acetate & $10198-23-9$ \\
\hline Acetaldehyde* & $75-07-0$ \\
\hline 1,2,3,6-Tetramethylbicyclo[2.2.2]octane & $62338-45-8$ \\
\hline 2,6-Di-tert-butyl-4-methylphenol & $128-37-0$ \\
\hline (-)-menthyl benzoate & $6284-35-1$ \\
\hline 3,3,6-Trimethyldecane & $62338-14-1$ \\
\hline 2,2,6,6-Tetramethylheptane & $40117-45-1$ \\
\hline 2-Methyl-1-butene & $563-46-2$ \\
\hline Hexyl methyl ether & $4747-07-3$ \\
\hline 1-Octen-3-yl-acetate & $2442-10-6$ \\
\hline 2-(4-tert-Butylbenzyl)propionaldehyde & $80-54-6$ \\
\hline Sabinene & $3387-41-5$ \\
\hline 2-Methyl-1-propene & $115-11-7$ \\
\hline alpha-Phellandrene & $99-83-2$ \\
\hline Isovaleraldehyde & $590-86-3$ \\
\hline 1-Vinylheptanol & $21964-44-3$ \\
\hline beta-Ionone & $14901-07-6$ \\
\hline 1-Hexanol & $111-27-3$ \\
\hline
\end{tabular}

*Compound classified as potentially hazardous 


\section{Car hanging air freshener}

\begin{tabular}{|c|c|}
\hline Compounds & CAS\# \\
\hline alpha-Pinene & $80-56-8$ \\
\hline Limonene & $138-86-3$ \\
\hline 3-Carene* & $13466-78-9$ \\
\hline beta-Pinene & $127-91-3$ \\
\hline Camphene & $79-92-5$ \\
\hline Bornyl acetate & $76-49-3$ \\
\hline 6-[(1E)-1-Propenyl]bicyclo[3.1.0]hexan-2-one & $75283-46-4$ \\
\hline Isobornyl acetate & $125-12-2$ \\
\hline beta-Phellandrene & $555-10-2$ \\
\hline Linalool & $78-70-6$ \\
\hline Tricyclene & $508-32-7$ \\
\hline Eucalyptol & $470-82-6$ \\
\hline Linalyl acetate & $115-95-7$ \\
\hline alpha-Terpineol & $98-55-5$ \\
\hline 2,7-Dimethyl-1-octanol & $15250-22-3$ \\
\hline $\begin{array}{l}\text { 3-Penten-2-one, 4-(2,6,6-trimethyl-2-cyclohexen- } \\
\text { 1-yl) }\end{array}$ & $114933-28-7$ \\
\hline Acetone & $67-64-1$ \\
\hline 1,3,3-Trimethylbicyclo[2.2.1]hept-2-yl acetate & $76109-40-5$ \\
\hline$(+)$-Camphor & $464-49-3$ \\
\hline gamma-Terpineol & $586-81-2$ \\
\hline Terpinolene & $586-62-9$ \\
\hline iso-Bornyl methacrylate & $7534-94-3$ \\
\hline Acetaldehyde* & $75-07-0$ \\
\hline Methanol* & $67-56-1$ \\
\hline beta-Terpinyl acetate & $10198-23-9$ \\
\hline 2-Phenethyl acetate & $103-45-7$ \\
\hline alpha-Phellandrene & $99-83-2$ \\
\hline 4-Carene & $29050-33-7$ \\
\hline Benzyl alcohol & $100-51-6$ \\
\hline Sabinene & $3387-41-5$ \\
\hline Piperonal & $120-57-0$ \\
\hline p-Anisaldehyde & $123-11-5$ \\
\hline Geranyl acetate & $105-87-3$ \\
\hline Neryl acetate & $141-12-8$ \\
\hline Propanal* & $123-38-6$ \\
\hline 1-Methyl-alpha-ionone & $7779-30-8$ \\
\hline Isopropyl alcohol & $67-63-0$ \\
\hline 3,7-Dimethyl-1-octanol & $106-21-8$ \\
\hline Ethanol & $64-17-5$ \\
\hline (Z)-sabinene hydrate & $15537-55-0$ \\
\hline beta-Terpineol & $138-87-4$ \\
\hline Phenethyl butyrate & $103-52-6$ \\
\hline$(-)$ - $\alpha$-Cedrene & $469-61-4$ \\
\hline N-butylbenzenesulfonamide & $3622-84-2$ \\
\hline gamma-Terpinene & $99-85-4$ \\
\hline 1-Butanol & $71-36-3$ \\
\hline
\end{tabular}




\begin{tabular}{ll}
\hline Caryophyllene & $87-44-5$ \\
\hline 2-Methyl-3-(p-isopropylphenyl)propionaldehyde & $103-95-7$ \\
\hline 2-Carene & $554-61-0$ \\
\hline Ethyl butyrate & $105-54-4$ \\
\hline Toluene* & $108-88-3$ \\
\hline 1-Terpinenol & $586-82-3$ \\
\hline Octanal & $124-13-0$ \\
\hline Cyclopropylidenecyclohexane & $14114-06-8$ \\
\hline Thujopsene & $470-40-6$ \\
\hline 2-Methyl-1-propene & $115-11-7$ \\
\hline Butylbenzene & $104-51-8$ \\
\hline Hexanal & $66-25-1$ \\
\hline 1,5-Dimethyl-1,4-cyclohexadiene & $4190-06-1$ \\
\hline Bicyclopentyl & $1636-39-1$ \\
\hline Tetracarbonylnickel* & $13463-39-3$ \\
\hline 2-methylundecanal & $110-41-8$ \\
\hline
\end{tabular}

*Compound classified as potentially hazardous 
7. Car vent air freshener ${ }^{N}$

\begin{tabular}{|c|c|}
\hline Compounds & CAS\# \\
\hline Limonene & $138-86-3$ \\
\hline Benzyl acetate & $140-11-4$ \\
\hline Allyl hexanoate & $123-68-2$ \\
\hline p-Anisaldehyde & $123-11-5$ \\
\hline Allyl heptanoate & $142-19-8$ \\
\hline gamma-Nonanolactone & $104-61-0$ \\
\hline Dihydromyrcenol & $18479-58-8$ \\
\hline Ethyl 2-methylpentanoate & $39255-32-8$ \\
\hline 2,6-Dimethyl octane & $2051-30-1$ \\
\hline 2,9-Dimethylundecane & $17301-26-7$ \\
\hline 3-Methyl-5-propylnonane & $31081-18-2$ \\
\hline Isoamyl acetate & $123-92-2$ \\
\hline 2-Isopropyl-5-methylhexanol & $2051-33-4$ \\
\hline 3,8-Dimethyldecane & $17312-55-9$ \\
\hline Heptadecyl acetate & $822-20-8$ \\
\hline 3,6-Dimethylundecane & $17301-28-9$ \\
\hline$\gamma$-Decalactone & $706-14-9$ \\
\hline Terpinolene & $586-62-9$ \\
\hline gamma-Terpinene & $99-85-4$ \\
\hline 5-Ethyl-2,2,3-trimethylheptane & $62199-06-8$ \\
\hline 2,6-Dimethyl-5-heptenal & $106-72-9$ \\
\hline 3,3,5-Trimethylheptane & $7154-80-5$ \\
\hline cis-3-Hexene-1-ol & $928-96-1$ \\
\hline 2,5-Dimethylundecane & $17301-22-3$ \\
\hline beta-Myrcene* & $123-35-3$ \\
\hline 2-methyl-6-methylene-7-octen-2-ol & 14314-21-7 \\
\hline Eucalyptol & $470-82-6$ \\
\hline Linalool & $78-70-6$ \\
\hline 2,2-Dimethyloctane & $15869-87-1$ \\
\hline 3,3,8-Trimethyldecane & $62338-16-3$ \\
\hline beta-Pinene & $127-91-3$ \\
\hline 2,2,4,6,6-Pentamethylheptane & $13475-82-6$ \\
\hline Allyl cyclohexanepropionate & $2705-87-5$ \\
\hline 2,2,3,3,5,6,6-Heptamethylheptane & $7225-67-4$ \\
\hline 1-Iododecane & $2050-77-3$ \\
\hline 3-Methyltetradecane & $18435-22-8$ \\
\hline 4,4'-Isopropylidenebis(2,6-dimethylphenol) & $5613-46-7$ \\
\hline cis-3-Hexenyl methoxy formate & $67633-96-9$ \\
\hline 3,9-Dimethylundecane & $17301-31-4$ \\
\hline 2-(4-tert-Butylbenzyl)propionaldehyde & $80-54-6$ \\
\hline 2-Methyl-5-propylnonane & $31081-17-1$ \\
\hline 2H-1-Benzopyran-2-one & $91-64-5$ \\
\hline 4,4-Dimethylundecane & $17312-68-4$ \\
\hline 2-Methylbutyl acetate & $624-41-9$ \\
\hline Benzyl alcohol & $100-51-6$ \\
\hline Piperonal & $120-57-0$ \\
\hline 2,2,11,11-Tetramethyldodecane & $127204-12-0$ \\
\hline
\end{tabular}




\begin{tabular}{ll}
\hline $\begin{array}{l}\text { 2,2-Dimethyltetradecane } \\
\text { Cyclopropane, 1,1-dimethyl-2-(3-methyl-1,3- }\end{array}$ & $59222-86-5$ \\
\hline butadienyl)- & $68998-21-0$ \\
\hline Hexyl salicylate & $6259-76-3$ \\
\hline 3-Methylundecane & $1002-43-3$ \\
\hline 1,4-Cineole & $470-67-7$ \\
\hline 6-Methyltridecane & $13287-21-3$ \\
\hline beta-trans-Ocimene & $3779-61-1$ \\
\hline 3,3,6-Trimethyldecane & $62338-14-1$ \\
\hline Allyl alcohol & $107-18-6$ \\
\hline 3,4,5,6-Tetramethyloctane & $62185-21-1$ \\
\hline Sabinene & $3387-41-5$ \\
\hline 2,2,3,4-Tetramethylpentane & $1186-53-4$ \\
\hline 6-Methyl-5-hepten-2-one & $110-93-0$ \\
\hline Acetaldehyde* & $75-07-0$ \\
\hline Decanal & $112-31-2$ \\
\hline Octanal & $124-13-0$ \\
\hline Methyl dihydrojasmonate & $24851-98-7$ \\
\hline 4-Ethyl-2,2,6,6-tetramethylheptane & $62108-31-0$ \\
\hline *Compound classified as potentially hazardous & \\
$N$ & \\
\hline
\end{tabular}




\section{Car vent air freshener}

\begin{tabular}{|c|c|}
\hline Compounds & CAS\# \\
\hline 3,5,5-Trimethylhexyl acetate & $58430-94-7$ \\
\hline Benzyl acetate & $140-11-4$ \\
\hline Dihydromyrcenol & $18479-58-8$ \\
\hline 4-tert-Butylcyclohexyl acetate & $32210-23-4$ \\
\hline Limonene & $138-86-3$ \\
\hline p-tert-Butyl cyclohexyl-acetate cis & $10411-92-4$ \\
\hline Linalool & $78-70-6$ \\
\hline 4-tert-Butylcyclohexanol & $98-52-2$ \\
\hline Ethyl 2-methylbutyrate & $7452-79-1$ \\
\hline Ethyl 2-methylpentanoate & $39255-32-8$ \\
\hline Acetaldehyde* & $75-07-0$ \\
\hline Glutaric acid dimethyl ester & $1119-40-0$ \\
\hline p-Anisaldehyde & $123-11-5$ \\
\hline Verdyl acetate & $5413-60-5$ \\
\hline Phenylethyl alcohol & $60-12-8$ \\
\hline (1R)-(-)-Nopol benzyl ether & 74851-17-5 \\
\hline Ethanol & $64-17-5$ \\
\hline$(\mathrm{R})-(+)-\beta$-Citronellol & $1117-61-9$ \\
\hline 2-tert-Butylcyclohexanol & $13491-79-7$ \\
\hline 2-Methoxynaphthalene & $93-04-9$ \\
\hline Isoamyl acetate & $123-92-2$ \\
\hline 2,6-Dimethyl-5-heptenal & $106-72-9$ \\
\hline Methyl phenylcarbinyl acetate & $93-92-5$ \\
\hline alpha-Terpineol & $98-55-5$ \\
\hline cis-Pinocampheol & $473-61-0$ \\
\hline Dimethyl adipate & $627-93-0$ \\
\hline 4-Methylanisole & $104-93-8$ \\
\hline cis- $\alpha$-Bisabolene & $29837-07-8$ \\
\hline 3,5,5-Trimethyl-1-hexanol & $3452-97-9$ \\
\hline $\begin{array}{l}\text { 3-Penten-2-one, 4-(2,6,6-trimethyl-2-cyclohexen- } \\
\text { 1-yl) }\end{array}$ & $114933-28-7$ \\
\hline$\alpha, \alpha$-Dimethylphenethyl acetate & $151-05-3$ \\
\hline Decanal & $112-31-2$ \\
\hline 3-Phenylbutyraldehyde & $16251-77-7$ \\
\hline 1,2,3,4-tetrahydro-1,4-methanonaphthalene & $4486-29-7$ \\
\hline Methyl benzoate & $93-58-3$ \\
\hline $\begin{array}{l}\text { Acetylmethylcyclohexene,4-acetyl-1-methyl-1- } \\
\text { cyclohexene }\end{array}$ & $70286-20-3$ \\
\hline alpha-Ionone & $127-41-3$ \\
\hline Acetone & $67-64-1$ \\
\hline 4,4'-Isopropylidenebis(2,6-dimethylphenol) & $5613-46-7$ \\
\hline 2-Methylbutyl acetate & $624-41-9$ \\
\hline Isoamyl butylate & $106-27-4$ \\
\hline Methanol* & $67-56-1$ \\
\hline Cyclofenchene & $488-97-1$ \\
\hline Camphene & $79-92-5$ \\
\hline beta-Myrcene* & $123-35-3$ \\
\hline
\end{tabular}




\begin{tabular}{ll}
\hline Dodecanal & $112-54-9$ \\
\hline 6-Methyl-5-hepten-2-one & $110-93-0$ \\
\hline Carane & $554-59-6$ \\
\hline 4-tert-Butylcyclohexene & $2228-98-0$ \\
\hline 2,5,5-Trimethyl-1-hexene & $62185-56-2$ \\
\hline Caryophyllene & $87-44-5$ \\
\hline (Z)-rose oxide & $16409-43-1$ \\
\hline 2-Methyl-1-propene & $115-11-7$ \\
\hline beta-Ionone & $14901-07-6$ \\
\hline Neryl acetate & $141-12-8$
\end{tabular}

*Compound classified as potentially hazardous 


\section{Car vent air freshener}

\begin{tabular}{|c|c|}
\hline Compounds & CAS\# \\
\hline Dihydromyrcenol & $18479-58-8$ \\
\hline Linalool & $78-70-6$ \\
\hline 3,5,5-Trimethylhexyl acetate & $58430-94-7$ \\
\hline Benzyl acetate & $140-11-4$ \\
\hline 3,4-Dimethylhex-4-en-2-one & $53252-21-4$ \\
\hline Benzyl propionate & $122-63-4$ \\
\hline Linalyl acetate & $115-95-7$ \\
\hline $\begin{array}{l}\text { (Z+E)-2-methyl-2-(4-methyl-3-pentenyl) } \\
\text { cyclopropane carbaldehyde }\end{array}$ & $97231-35-1$ \\
\hline 2-Acetylcyclopentanone & $1670-46-8$ \\
\hline Prenyl acetate & $1191-16-8$ \\
\hline 3,7-Dimethyl-6-octenoic acid & $502-47-6$ \\
\hline 4-tert-Butylcyclohexyl acetate & $32210-23-4$ \\
\hline Verdyl acetate & $5413-60-5$ \\
\hline 2-Cyclohexene-1-Acetaldehyde, 3-methyl- & $129993-40-4$ \\
\hline Menthyl acetate & $89-48-5$ \\
\hline Isomenthol acetate & $20777-45-1$ \\
\hline Ethanol & $64-17-5$ \\
\hline (1R)-(-)-Nopol benzyl ether & $74851-17-5$ \\
\hline 2-Isopropyl-5-methylcyclohexyl acetate & $20777-36-0$ \\
\hline Methyl benzoate & $93-58-3$ \\
\hline DL-menthyl acetate & $16409-45-3$ \\
\hline $\begin{array}{l}\text { Acetylmethylcyclohexene,4-acetyl-1-methyl-1- } \\
\text { cyclohexene }\end{array}$ & $70286-20-3$ \\
\hline 1,5-Diethyl-2,3-dimethylcyclohexane & 74663-66-4 \\
\hline Limonene & $138-86-3$ \\
\hline 3,4,5-Trimethyl-1-hexene & $56728-10-0$ \\
\hline p-tert-Butyl cyclohexyl-acetate cis & $10411-92-4$ \\
\hline 3-Ethyl-2-hexene & $620-00-8$ \\
\hline 1-tert-Butoxy-3-Methylcyclohexene & $40648-24-6$ \\
\hline 1-tert-Butoxy-3-Methylcyclohexene & $40648-24-6$ \\
\hline $\begin{array}{l}\text { 3-Penten-2-one, 4-(2,6,6-trimethyl-2-cyclohexen- } \\
\text { 1-yl) }\end{array}$ & $114933-28-7$ \\
\hline$(Z)$-rose oxide & $16409-43-1$ \\
\hline Acetaldehyde* & $75-07-0$ \\
\hline 7-Methyl-3-(1-methylethyl)-1,5-octadiene & $74630-12-9$ \\
\hline Phenyl cyclobutanecarboxylate & $30466-31-0$ \\
\hline 2,3-Dimethyl-2,3-diphenylbutane & $1889-67-4$ \\
\hline 2,5-Dimethyl-2-undecene & $49622-16-4$ \\
\hline 2-methylundecanal & $110-41-8$ \\
\hline Acetone & $67-64-1$ \\
\hline 4-Ethyl-2-hexene & $19780-46-2$ \\
\hline Dicyclopentenyl alcohol & $37275-49-3$ \\
\hline 1,2,3,4-tetrahydro-1,4-methanonaphthalene & $4486-29-7$ \\
\hline Bornyl acetate & $76-49-3$ \\
\hline alpha-Terpineol & $98-55-5$ \\
\hline 2-methyl-dec-3-en-5-one & $32064-75-8$ \\
\hline
\end{tabular}




\begin{tabular}{ll}
\hline 2,5,5-Trimethyl-1-hexene & $62185-56-2$ \\
\hline Isopropyl alcohol & $67-63-0$ \\
\hline 2-Methyl-4-propyl-1,3-oxathiane & $67715-80-4$ \\
\hline 1-Methyl-alpha-ionone & $7779-30-8$ \\
\hline beta-Ionone & $14901-07-6$ \\
\hline Nonyl 2-methylpropanoate & $10522-34-6$ \\
\hline 2-Methoxynaphthalene & $93-04-9$ \\
\hline 2-Methyl-1-propene & $115-11-7$ \\
\hline Isopentane & $78-78-4$ \\
\hline Neryl acetate & $141-12-8$
\end{tabular}

*Compound classified as potentially hazardous 


\section{Car vent air freshener}

\begin{tabular}{ll}
\hline Compounds & CAS\# \\
\hline Limonene & $138-86-3$ \\
\hline Dihydromyrcenol & $18479-58-8$ \\
\hline Glutaric acid dimethyl ester & $1119-40-0$ \\
\hline Linalool & $78-70-6$ \\
\hline Dimethyl adipate & $627-93-0$ \\
\hline Eucalyptol & $470-82-6$ \\
\hline Ethanol & $64-17-5$ \\
\hline Linalyl acetate & $115-95-7$ \\
\hline Methanol* & $67-56-1$ \\
\hline beta-Myrcene* & $123-35-3$ \\
\hline (+)-Camphor & $464-49-3$ \\
\hline alpha-Terpineol & $98-55-5$ \\
\hline Cedrol & $77-53-2$ \\
\hline beta-Citral & $106-26-3$ \\
\hline 3-Penten-2-one, 4-(2,6,6-trimethyl-2-cyclohexen- & $114933-28-7$ \\
1-yl) & \\
\hline Allyl (isopentyloxy)acetate & $67634-00-8$ \\
\hline Isobornyl acetate & $125-12-2$ \\
\hline gamma-Terpineol & $586-81-2$ \\
\hline 2-Isopropenyl-5-methylhex-4-enal & $75697-98-2$ \\
\hline 1-Isopropyl-2-methyl-3-(1- & $24524-51-4$ \\
methylethylidene)cyclopropane & \\
\hline Caryophyllene & $87-44-5$ \\
\hline Acetone & $67-64-1$ \\
\hline beta-Phellandrene & $555-10-2$ \\
\hline alpha-Pinene & $80-56-8$ \\
\hline beta-Terpinyl acetate & $10198-23-9$ \\
\hline 3-Isopropyl-5-methyl-4-hexen-2-one & $77142-85-9$ \\
\hline Sabinene & $3387-41-5$ \\
\hline 2,9-Dimethylundecane & $17301-26-7$ \\
\hline Ethyl safranate & $35044-59-8$ \\
\hline Allyl butyrate & $2051-78-7$ \\
\hline Citronellyl formate & $105-85-1$ \\
\hline 2,4-Dimethylheptane & $2213-23-2$ \\
\hline 3-Carene* & $13466-78-9$ \\
\hline Dimethyl succinate & $106-65-0$ \\
\hline Octanal & $124-13-0$ \\
\hline beta-Terpineol & $138-87-4$ \\
\hline 2,4-Dimethyl-1-heptene & $19549-87-2$ \\
\hline Neryl acetate & $141-12-8$ \\
\hline 1,3-Hexadiene,3-ethyl-2,5-dimethyl & $62338-07-2$ \\
\hline 2,3,3-Trimethylpentane & $560-21-4$ \\
\hline 1-Methyl-alpha-ionone & $7779-30-8$ \\
\hline 2-Methyl-1-pentene & $763-29-1$ \\
\hline trans-2-Octene & $13389-42-9$ \\
\hline beta-trans-Ocimene & $3779-61-1$ \\
\hline 2-Methylpentane & $107-83-5$ \\
\hline & \\
\hline
\end{tabular}




\begin{tabular}{ll}
\hline 1-Octene & $111-66-0$ \\
\hline Borneol & $10385-78-1$ \\
\hline 4-Methyloctane & $2216-34-4$ \\
\hline 1,1-Dimethylallyl alcohol & $115-18-4$ \\
\hline 4-methylheptane & $589-53-7$ \\
\hline (+)-trans,trans-5-Caranol & $6909-22-4$ \\
\hline (E)-3,3-DimethylcyclohexylideneAcetaldehyde & $26532-25-2$ \\
\hline Acetaldehyde* & $75-07-0$ \\
\hline 2-Octene & $111-67-1$ \\
\hline 7-Hydroxycitronellal & $107-75-5$ \\
\hline trans-4-Octene & $14850-23-8$ \\
\hline
\end{tabular}

*Compound classified as potentially hazardous 


\section{Car vent air freshener ${ }^{N}$}

\begin{tabular}{|c|c|}
\hline Compounds & CAS\# \\
\hline Ethanol & $64-17-5$ \\
\hline Limonene & $138-86-3$ \\
\hline Benzyl alcohol & $100-51-6$ \\
\hline Ethyl 2-methylbutyrate & $7452-79-1$ \\
\hline Hexyl acetate & $142-92-7$ \\
\hline Ethyl butyrate & $105-54-4$ \\
\hline Isoamyl acetate & $123-92-2$ \\
\hline Linalool & $78-70-6$ \\
\hline Methyl phenylcarbinyl acetate & $93-92-5$ \\
\hline Dihydromyrcenol & $18479-58-8$ \\
\hline D,L-isobornyl acetate & $92618-89-8$ \\
\hline Ethyl hexanoate & $123-66-0$ \\
\hline Verdyl acetate & $5413-60-5$ \\
\hline Benzyl acetate & $140-11-4$ \\
\hline Allyl hexanoate & $123-68-2$ \\
\hline Benzaldehyde & $100-52-7$ \\
\hline 2-Methyl-3-(p-isopropylphenyl)propionaldehyde & $103-95-7$ \\
\hline 2-Methylbutyl acetate & $624-41-9$ \\
\hline Phenylethyl alcohol & $60-12-8$ \\
\hline Eucalyptol & $470-82-6$ \\
\hline Ethyl 2-methyl-1,3-dioxolane-2-acetate & $6413-10-1$ \\
\hline (4E)-4-Hexenyl acetate & $72237-36-6$ \\
\hline Benzyl propionate & $122-63-4$ \\
\hline PhenylAcetaldehyde dimethyl acetal & $101-48-4$ \\
\hline 4-tert-Butylcyclohexyl acetate & $32210-23-4$ \\
\hline$\alpha, \alpha$-Dimethylphenethyl acetate & $151-05-3$ \\
\hline gamma-Nonanolactone & $104-61-0$ \\
\hline alpha-Pinene & $80-56-8$ \\
\hline p-tert-Butyl cyclohexyl-acetate cis & $10411-92-4$ \\
\hline cis-3-Hexene-1-ol & $928-96-1$ \\
\hline Linalyl acetate & $115-95-7$ \\
\hline Allyl (isopentyloxy)acetate & $67634-00-8$ \\
\hline 2-(phenylmethyl)-1,3-dioxolane & $101-49-5$ \\
\hline$(-)$ - $\alpha$-Cedrene & $469-61-4$ \\
\hline cis-1,2-Dimethylcyclopropane & $930-18-7$ \\
\hline Methanol* & $67-56-1$ \\
\hline 2-Methyl-2-propanol & $75-65-0$ \\
\hline Allyl cyclohexanepropionate & $2705-87-5$ \\
\hline Allyl heptanoate & $142-19-8$ \\
\hline Neryl acetate & $141-12-8$ \\
\hline Sabinene & $3387-41-5$ \\
\hline 2-tert-Butylcyclohexanol & $13491-79-7$ \\
\hline beta-Myrcene* & $123-35-3$ \\
\hline 4,4'-Isopropylidenebis(2,6-dimethylphenol) & $5613-46-7$ \\
\hline $\begin{array}{l}\text { 3-Penten-2-one, 4-(2,6,6-trimethyl-2-cyclohexen- } \\
\text { 1-yl) }\end{array}$ & $114933-28-7$ \\
\hline 1-Isopropyl-2-methyl-3-(1- & $24524-51-4$ \\
\hline
\end{tabular}




\begin{tabular}{ll}
\hline methylethylidene)cyclopropane & \\
\hline Methyl dihydrojasmonate & $24851-98-7$ \\
\hline Acetone & $67-64-1$ \\
\hline 1,3-Hexadiene,3-ethyl-2,5-dimethyl & $62338-07-2$ \\
\hline Allyl alcohol & $107-18-6$ \\
\hline L-menthol & $2216-51-5$ \\
\hline Ethyl acetate & $141-78-6$ \\
\hline 2-Phenoxyethyl isobutyrate & $103-60-6$ \\
\hline 2-Methyl-1-propene & $115-11-7$ \\
\hline *Compound classified as potentially hazardous \\
${ }^{N}$ Product with the claim of being "natural" or a related term.
\end{tabular}




\section{Car vent air freshener}

\begin{tabular}{ll}
\hline Compounds & CAS\# \\
\hline Tetrahydrolinalool & $57706-88-4$ \\
\hline Benzyl acetate & $140-11-4$ \\
\hline Isoamyl butylate & $106-27-4$ \\
\hline Linalool & $78-70-6$ \\
\hline Methyl phenylcarbinyl acetate & $93-92-5$ \\
\hline Limonene & $138-86-3$ \\
\hline Hexyl acetate & $142-92-7$ \\
\hline Allyl hexanoate & $123-68-2$ \\
\hline Ethyl heptanoate & $106-30-9$ \\
\hline Dihydromyrcenol & $18479-58-8$ \\
\hline 4-tert-Butylcyclohexyl acetate & $32210-23-4$ \\
\hline Phenylethyl alcohol & $60-12-8$ \\
\hline Allyl heptanoate & $142-19-8$ \\
\hline 2,6-Di-tert-butyl-4-methylphenol & $128-37-0$ \\
\hline Verdyl acetate & $5413-60-5$ \\
\hline Isozonarol & $39707-55-6$ \\
\hline Benzaldehyde & $100-52-7$ \\
\hline 2-tert-Butylcyclohexanol & $13491-79-7$ \\
\hline Allyl (isopentyloxy)acetate & $67634-00-8$ \\
\hline Diphenyl ether & $101-84-8$ \\
\hline Methyl benzoate & $93-58-3$ \\
\hline p-Anisaldehyde & $123-11-5$ \\
\hline Allyl 2-ethyl butyrate & $7493-69-8$ \\
\hline 2-Methylbutyl acetate & $624-41-9$ \\
\hline 1,2-Dihydrolinalool & $18479-51-1$ \\
\hline Isoamyl acetate & $123-92-2$ \\
\hline Acetaldehyde* & $75-07-0$ \\
\hline
\end{tabular}

*Compound classified as potentially hazardous 
Supplementary Table 2: All emitted VOCs from all of the car air fresheners studied

\begin{tabular}{|c|c|c|c|c|}
\hline \multirow[t]{2}{*}{ Compound } & \multirow[t]{2}{*}{ CAS \# } & \multicolumn{3}{|c|}{ Prevalence (\# of Products) } \\
\hline & & Total & $\begin{array}{l}\text { Regular } \\
(\mathrm{n}=8)\end{array}$ & $\begin{array}{l}\text { Natural } \\
(\mathrm{n}=4)\end{array}$ \\
\hline Limonene & $138-86-3$ & 10 & 8 & 2 \\
\hline Benzyl acetate & $140-11-4$ & 9 & 5 & 4 \\
\hline Acetone & $67-64-1$ & 9 & 7 & 2 \\
\hline Ethanol & $64-17-5$ & 9 & 6 & 3 \\
\hline Linalool & $78-70-6$ & 8 & 6 & 2 \\
\hline 2-Methylbutyl acetate & $624-41-9$ & 8 & 4 & 4 \\
\hline Acetaldehyde* & $75-07-0$ & 8 & 7 & 1 \\
\hline Methanol* & $67-56-1$ & 8 & 5 & 3 \\
\hline $\begin{array}{l}\text { 3-Penten-2-one, 4-(2,6,6- } \\
\text { trimethyl-2-cyclohexen-1-yl) }\end{array}$ & $114933-28-7$ & 7 & 4 & 3 \\
\hline beta-Myrcene* & $123-35-3$ & 7 & 5 & 2 \\
\hline Dihydromyrcenol & $18479-58-8$ & 7 & 5 & 2 \\
\hline Isoamyl acetate & $123-92-2$ & 7 & 4 & 3 \\
\hline Neryl acetate & $141-12-8$ & 7 & 6 & 1 \\
\hline 4-tert-Butylcyclohexyl acetate & $32210-23-4$ & 6 & 4 & 2 \\
\hline Eucalyptol & $470-82-6$ & 6 & 4 & 2 \\
\hline Sabinene & $3387-41-5$ & 6 & 4 & 2 \\
\hline Verdyl acetate & $5413-60-5$ & 6 & 5 & 1 \\
\hline 2-Methyl-1-propene & $115-11-7$ & 5 & 4 & 1 \\
\hline alpha-Pinene & $80-56-8$ & 5 & 4 & 1 \\
\hline alpha-Terpineol & $98-55-5$ & 5 & 5 & 0 \\
\hline beta-Pinene & $127-91-3$ & 5 & 4 & 1 \\
\hline Ethyl butyrate & $105-54-4$ & 5 & 2 & 3 \\
\hline Linalyl acetate & $115-95-7$ & 5 & 4 & 1 \\
\hline p-Anisaldehyde & $123-11-5$ & 5 & 4 & 1 \\
\hline $\begin{array}{l}\text { p-tert-Butyl cyclohexyl-acetate } \\
\text { cis }\end{array}$ & $10411-92-4$ & 5 & 3 & 2 \\
\hline 1-Methyl-alpha-ionone & $7779-30-8$ & 4 & 3 & 1 \\
\hline Allyl heptanoate & $142-19-8$ & 4 & 2 & 2 \\
\hline Allyl hexanoate & $123-68-2$ & 4 & 2 & 2 \\
\hline Benzaldehyde & $100-52-7$ & 4 & 1 & 3 \\
\hline Camphene & $79-92-5$ & 4 & 4 & 0 \\
\hline Isopropyl alcohol & $67-63-0$ & 4 & 4 & 0 \\
\hline Methyl phenylcarbinyl acetate & $93-92-5$ & 4 & 3 & 1 \\
\hline o-Cymene & $527-84-4$ & 4 & 4 & 0 \\
\hline $\begin{array}{l}\text { 2,6-Di-tert-butyl-4- } \\
\text { methylphenol }\end{array}$ & $128-37-0$ & 3 & 3 & 0 \\
\hline
\end{tabular}




\begin{tabular}{|c|c|c|c|}
\hline 2,9-Dimethylundecane & $17301-26-7$ & 3 & 2 \\
\hline 2-tert-Butylcyclohexanol & $13491-79-7$ & 3 & 2 \\
\hline $\begin{array}{l}\text { 4,4'-Isopropylidenebis }(2,6- \\
\text { dimethylphenol) }\end{array}$ & $5613-46-7$ & 5 & 2 \\
\hline Allyl (isopentyloxy)acetate & $67634-00-8$ & 3 & 2 \\
\hline Allyl alcohol & $107-18-6$ & 3 & 1 \\
\hline Allyl cyclohexanepropionate & $2705-87-5$ & 3 & 1 \\
\hline Benzyl alcohol & $100-51-6$ & 3 & 1 \\
\hline beta-Ionone & $14901-07-6$ & 3 & 3 \\
\hline beta-Phellandrene & $555-10-2$ & 3 & 3 \\
\hline beta-Terpinyl acetate & $10198-23-9$ & 3 & 3 \\
\hline Caryophyllene & $87-44-5$ & 3 & 3 \\
\hline gamma-Nonanolactone & $104-61-0$ & 3 & 1 \\
\hline gamma-Terpinene & $99-85-4$ & 3 & 2 \\
\hline gamma-Terpineol & $586-81-2$ & 3 & 3 \\
\hline Isoamyl butylate & $106-27-4$ & 3 & 3 \\
\hline Isobornyl acetate & $125-12-2$ & 3 & 3 \\
\hline Methyl benzoate & $93-58-3$ & 3 & 3 \\
\hline N-butylbenzenesulfonamide & $3622-84-2$ & 3 & 2 \\
\hline Octanal & $124-13-0$ & 3 & 2 \\
\hline Phenylethyl alcohol & $60-12-8$ & 3 & 2 \\
\hline Terpinolene & $586-62-9$ & 3 & 2 \\
\hline$(-)$ - $\alpha$-Cedrene & $469-61-4$ & 2 & 1 \\
\hline (+)-Camphor & $464-49-3$ & 2 & 2 \\
\hline (1R)-(-)-Nopol benzyl ether & $74851-17-5$ & 2 & 2 \\
\hline (Z)-rose oxide & $16409-43-1$ & 2 & 2 \\
\hline $\begin{array}{l}\text { 1,2,3,4-tetrahydro-1,4- } \\
\text { methanonaphthalene }\end{array}$ & $4486-29-7$ & 2 & 2 \\
\hline $\begin{array}{l}\text { 1,3-Hexadiene,3-ethyl-2,5- } \\
\text { dimethyl }\end{array}$ & $62338-07-2$ & 2 & 1 \\
\hline $\begin{array}{l}\text { 1-Isopropyl-2-methyl-3-(1- } \\
\text { methylethylidene)cyclopropan } \\
\text { e }\end{array}$ & $24524-51-4$ & 2 & 1 \\
\hline $\begin{array}{l}\text { 1-tert-Butoxy-3- } \\
\text { Methylcyclohexene }\end{array}$ & $40648-24-6$ & 2 & 2 \\
\hline $\begin{array}{l}\text { 2-(4-tert- } \\
\text { Butylbenzyl)propionaldehyde }\end{array}$ & $80-54-6$ & 2 & 1 \\
\hline $\begin{array}{l}2,2,11,11- \\
\text { Tetramethyldodecane }\end{array}$ & $127204-12-0$ & 2 & 1 \\
\hline 2,2-Dimethyltetradecane & $59222-86-5$ & 2 & 1 \\
\hline 2,5,5-Trimethyl-1-hexene & $62185-56-2$ & 2 & 2 \\
\hline 2,5-Dimethylundecane & $17301-22-3$ & 2 & 1 \\
\hline 2,6-Dimethyl-5-heptenal & $106-72-9$ & 2 & 1 \\
\hline 2-Methoxynaphthalene & $93-04-9$ & 2 & 2 \\
\hline
\end{tabular}


2-Methyl-3-(p-

103-95-7

isopropylphenyl)propionaldeh

yde

2-Methylpentane

107-83-5

2

2-methylundecanal

$110-41-8$

2

3,3,6-Trimethyldecane

62338-14-1

3,5,5-Trimethylhexyl acetate

58430-94-7

2

3,8-Dimethyldecane

17312-55-9

13466-78-9

2

3-Carene*

3-Methyl-5-propylnonane

31081-18-2

$1002-43-3$

17312-68-4

4,4-Dimethylundecane

110-93-0

6-Methyl-5-hepten-2-one

13287-21-3

70286-20-3

Acetylmethylcyclohexene,4acetyl-1-methyl-1-cyclohexene alpha-Phellandrene

99-83-2

Amyl acetate

628-63-7

Benzyl propionate

$122-63-4$

beta-Citral

106-26-3

beta-Terpineol

138-87-4

3779-61-1

beta-trans-Ocimene

76-49-3

928-96-1

cis-3-Hexene-1-ol

cis-Ethyl 3-methyl-3-

19464-95-0

2

2

2

2

2

2

2

2

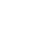

2

2

2

2

2

2

2

2

2

2

2

488-97-1

Cyclofenchene

D,L-isobornyl acetate

92618-89-8

112-31-2

Decanal

37275-49-3

627-93-0

106-65-0

7452-79-1

39255-32-8

141-78-6

123-66-0

1119-40-0

142-92-7

78-78-4

134-20-3

24851-98-7

529-20-4

120-57-0

2

2

2

2

2

2

2

2

2

2

2

2

2

2

Methyl anthranilate

Methyl dihydrojasmonate

o-Tolualdehyde

Piperonal

2

2

2

2

2
2
2
2
2
2
2
2
2
2
2
2




p-Tolualdehyde
trans-Ethyl 3-Methyl-3-
phenylglycidate
$\alpha, \alpha$-Dimethylphenethyl acetate
$\gamma$-Decalactone
(-)-menthyl benzoate
(+)-trans,trans-5-Caranol
(4E)-4-Hexenyl acetate
(E)-3,3-
Dimethylcyclohexylideneaceta
ldehyde

(E)-citral

(R)-(+)- $\beta$-Citronellol

104-87-0

19464-92-7 2

$19464-92-7-2$

151-05-3

706-14-9

6284-35-1

6909-22-4

72237-36-6

26532-25-2

$\begin{array}{lll}2 & 0 & 2 \\ 2 & 0 & 2 \\ 2 & 1 & 1 \\ 2 & 1 & 1 \\ 1 & 1 & 0 \\ 1 & 1 & 0 \\ 1 & 0 & 1 \\ 1 & 1 & 0\end{array}$

(Z)-sabinene hydrate

$141-27-5$

1117-61-9

0

$15537-55-0$

0

0

(Z+E)-2-methyl-2-(4-methyl-

97231-35-1

0

3-pentenyl) cyclopropane

carbaldehyde

1-(2-Methoxypropoxy)propan-

2-ol

1,1-Dimethylallyl alcohol

13429-07-7 1

0

$115-18-4$

1,2,3,6-

62338-45-8

0

0

Tetramethylbicyclo[2.2.2]octa ne

1,2-Dihydrolinalool

18479-51-1

76109-40-5

0

0

Trimethylbicyclo[2.2.1]hept-2yl acetate

1,4-Cineole

470-67-7

1,5-Diethyl-2,3-

74663-66-4

dimethylcyclohexane

1,5-Dimethyl-1,4-

4190-06-1

cyclohexadiene

Tricyclene

508-32-7

10-(oxan-2-yloxy)decan-1-ol

43047-93-4

10-Methylnonadecane

56862-62-5

1-Butanol

71-36-3

1-Hexanol

111-27-3

1-Iododecane

2050-77-3

2442-10-6

1-Octen-3-yl-acetate

111-66-0

71-41-0

1-Pentanol

71-23-8

1-Propanol

586-82-3

1-Terpinenol

21964-44-3

1
1

$\begin{array}{ll}1 & 0 \\ 1 & 0\end{array}$

1-Vinylheptanol

21964-44-3

1
1
1
1
1
1
1
1
1
1
1
1
1
1
1

$\begin{array}{ll}0 & 1 \\ 1 & 0 \\ 1 & 0 \\ 1 & 0 \\ 0 & 1 \\ 1 & 0 \\ 1 & 0 \\ 1 & 0 \\ 0 & 1 \\ 1 & 0 \\ 1 & 0 \\ 0 & 1 \\ 1 & 0 \\ 1 & 0 \\ 1 & 0\end{array}$




\begin{tabular}{|c|c|c|c|}
\hline $\begin{array}{l}\text { 2-(phenylmethyl)-1,3- } \\
\text { dioxolane }\end{array}$ & $101-49-5$ & 1 & 0 \\
\hline $\begin{array}{l}2,2,3,3,5,6,6- \\
\text { Heptamethylheptane }\end{array}$ & $7225-67-4$ & 1 & 0 \\
\hline 2,2,3,4-Tetramethylpentane & $1186-53-4$ & 1 & 0 \\
\hline 2,2,3-Trimethylnonane & $55499-04-2$ & 1 & 1 \\
\hline 2,2,4,6,6-Pentamethylheptane & $13475-82-6$ & 1 & 0 \\
\hline 2,2,4-Trimethylhexane & $16747-26-5$ & 1 & 1 \\
\hline 2,2,6,6-Tetramethylheptane & $40117-45-1$ & 1 & 1 \\
\hline 2,2,6-Trimethyldecane & $62237-97-2$ & 1 & 1 \\
\hline 2,2,7,7-Tetramethyloctane & $1071-31-4$ & 1 & 1 \\
\hline 2,2,9-Trimethyldecane & $62238-00-0$ & 1 & 1 \\
\hline 2,2-Dimethyloctane & $15869-87-1$ & 1 & 0 \\
\hline 2,3,3-Trimethylpentane & $560-21-4$ & 1 & 1 \\
\hline 2,3,6-Trimethyldecane & $62238-12-4$ & 1 & 1 \\
\hline $\begin{array}{l}\text { 2,3-Dimethyl-2,3- } \\
\text { diphenylbutane }\end{array}$ & $1889-67-4$ & 1 & 1 \\
\hline 2,4-Dimethyl-1-heptene & $19549-87-2$ & 1 & 1 \\
\hline 2,4-Dimethyl-3-pentanol & $600-36-2$ & 1 & 1 \\
\hline 2,4-Dimethylheptane & $2213-23-2$ & 1 & 1 \\
\hline 2,5-Dimethyl-2-undecene & 49622-16-4 & 1 & 1 \\
\hline 2,5-Dimethyltridecane & $56292-66-1$ & 1 & 1 \\
\hline 2,6,6-Trimethyloctane & $54166-32-4$ & 1 & 1 \\
\hline 2,6-Dimethyl octane & 2051-30-1 & 1 & 0 \\
\hline 2,7-Dimethyl-1-octanol & $15250-22-3$ & 1 & 1 \\
\hline 2-Acetylcyclopentanone & $1670-46-8$ & 1 & 1 \\
\hline 2-Bromotetradecane & $74036-95-6$ & 1 & 1 \\
\hline 2-Carene & $554-61-0$ & 1 & 1 \\
\hline $\begin{array}{l}\text { 2-Cyclohexene-1- } \\
\text { acetaldehyde, 3-methyl- }\end{array}$ & $129993-40-4$ & 1 & 1 \\
\hline 2H-1-Benzopyran-2-one & $91-64-5$ & 1 & 0 \\
\hline $\begin{array}{l}\text { 2-Isopropenyl-5-methylhex-4- } \\
\text { enal }\end{array}$ & $75697-98-2$ & 1 & 1 \\
\hline $\begin{array}{l}\text { 2-Isopropyl-5- } \\
\text { methylcyclohexyl acetate }\end{array}$ & $20777-36-0$ & 1 & 1 \\
\hline 2-Isopropyl-5-methylhexanol & $2051-33-4$ & 1 & 0 \\
\hline 2-Methyl-1-butene & $563-46-2$ & 1 & 1 \\
\hline 2-Methyl-1-pentene & $763-29-1$ & 1 & 1 \\
\hline 2-Methyl-2-propanol & $75-65-0$ & 1 & 0 \\
\hline $\begin{array}{l}\text { 2-Methyl-4-propyl-1,3- } \\
\text { oxathiane }\end{array}$ & $67715-80-4$ & 1 & 1 \\
\hline 2-Methyl-5-propylnonane & $31081-17-1$ & 1 & 0 \\
\hline $\begin{array}{l}\text { 2-methyl-6-methylene-7- } \\
\text { octen-2-ol }\end{array}$ & $14314-21-7$ & 1 & 0 \\
\hline
\end{tabular}




\begin{tabular}{|c|c|c|c|}
\hline 2-methyl-dec-3-en-5-one & $32064-75-8$ & 1 & 1 \\
\hline 2-Octene & $111-67-1$ & 1 & 1 \\
\hline 2-Phenethyl acetate & $103-45-7$ & 1 & 1 \\
\hline 2-Phenoxyethyl isobutyrate & $103-60-6$ & 1 & 0 \\
\hline 3,3,5-Trimethylheptane & $7154-80-5$ & 1 & 0 \\
\hline 3,3,8-Trimethyldecane & $62338-16-3$ & 1 & 0 \\
\hline 3,3-Dimethylundecane & $17312-65-1$ & 1 & 1 \\
\hline 3,4,5,6-Tetramethyloctane & $62185-21-1$ & 1 & 0 \\
\hline 3,4,5-Trimethyl-1-hexene & $56728-10-0$ & 1 & 1 \\
\hline 3,4-Dimethylhex-4-en-2-one & $53252-21-4$ & 1 & 1 \\
\hline 3,5,5-Trimethyl-1-hexanol & $3452-97-9$ & 1 & 1 \\
\hline 3,6-Dimethylundecane & $17301-28-9$ & 1 & 0 \\
\hline 3,7-Dimethyl-1-octanol & $106-21-8$ & 1 & 1 \\
\hline 3,7-Dimethyl-6-octenoic acid & $502-47-6$ & 1 & 1 \\
\hline 3,9-Dimethylundecane & $17301-31-4$ & 1 & 0 \\
\hline 3-Ethyl-2-hexene & $620-00-8$ & 1 & 1 \\
\hline $\begin{array}{l}\text { 3-Isopropyl-5-methyl-4-hexen- } \\
\text { 2-one }\end{array}$ & $77142-85-9$ & 1 & 1 \\
\hline 3-Methyltetradecane & $18435-22-8$ & 1 & 0 \\
\hline 3-Phenylbutyraldehyde & $16251-77-7$ & 1 & 1 \\
\hline 4,6-Dimethyldodecane & $61141-72-8$ & 1 & 1 \\
\hline 4-Carene & $29050-33-7$ & 1 & 1 \\
\hline $\begin{array}{l}\text { 4-Ethyl-2,2,6,6- } \\
\text { tetramethylheptane }\end{array}$ & $62108-31-0$ & 1 & 0 \\
\hline 4-Ethyl-2-hexene & $19780-46-2$ & 1 & 1 \\
\hline 4-Methylanisole & $104-93-8$ & 1 & 1 \\
\hline 4-methylheptane & $589-53-7$ & 1 & 1 \\
\hline 4-Methyloctane & $2216-34-4$ & 1 & 1 \\
\hline 4-Terpineol & $562-74-3$ & 1 & 1 \\
\hline 4-tert-Butylcyclohexanol & $98-52-2$ & 1 & 1 \\
\hline 4-tert-Butylcyclohexene & $2228-98-0$ & 1 & 1 \\
\hline 5-Ethyl-2,2,3-trimethylheptane & $62199-06-8$ & 1 & 0 \\
\hline $\begin{array}{l}\text { 6-[(1E)-1- } \\
\text { Propenyl]bicyclo[3.1.0]hexan- } \\
\text { 2-one }\end{array}$ & $75283-46-4$ & 1 & 1 \\
\hline 7-Hydroxycitronellal & $107-75-5$ & 1 & 1 \\
\hline $\begin{array}{l}\text { 7-Methyl-3-(1-methylethyl)- } \\
\text { 1,5-octadiene }\end{array}$ & $74630-12-9$ & 1 & 1 \\
\hline Allyl 2-ethyl butyrate & $7493-69-8$ & 1 & 1 \\
\hline Allyl butyrate & $2051-78-7$ & 1 & 1 \\
\hline alpha-Ionone & $127-41-3$ & 1 & 1 \\
\hline Bicyclopentyl & $1636-39-1$ & 1 & 1 \\
\hline Borneol & $10385-78-1$ & 1 & 1 \\
\hline
\end{tabular}




\begin{tabular}{|c|c|c|c|}
\hline Butane & $106-97-8$ & 1 & 1 \\
\hline Butylbenzene & $104-51-8$ & 1 & 1 \\
\hline Cedrol & $77-53-2$ & 1 & 1 \\
\hline cis-1,2-Dimethylcyclopropane & $930-18-7$ & 1 & 0 \\
\hline $\begin{array}{l}\text { cis-3-Hexenyl methoxy } \\
\text { formate }\end{array}$ & $67633-96-9$ & 1 & 0 \\
\hline cis-Pinocampheol & $473-61-0$ & 1 & 1 \\
\hline cis- $\alpha$-Bisabolene & $29837-07-8$ & 1 & 1 \\
\hline Citronellyl formate & $105-85-1$ & 1 & 1 \\
\hline $\begin{array}{l}\text { Cyclopropane, 1,1-dimethyl-2- } \\
\text { (3-methyl-1,3-butadienyl)- }\end{array}$ & $68998-21-0$ & 1 & 0 \\
\hline Cyclopropylidenecyclohexane & $14114-06-8$ & 1 & 1 \\
\hline Diethyl malonate & $105-53-3$ & 1 & 1 \\
\hline Diisopropyl ether & $108-20-3$ & 1 & 1 \\
\hline Dipentyl ether & $693-65-2$ & 1 & 1 \\
\hline Diphenyl ether & $101-84-8$ & 1 & 1 \\
\hline DL-menthyl acetate & $16409-45-3$ & 1 & 1 \\
\hline Dodecanal & $112-54-9$ & 1 & 1 \\
\hline $\begin{array}{l}\text { Ethyl 2-methyl-1,3-dioxolane- } \\
\text { 2-acetate }\end{array}$ & $6413-10-1$ & 1 & 0 \\
\hline Ethyl heptanoate & $106-30-9$ & 1 & 1 \\
\hline Ethyl isobutyrate & $97-62-1$ & 1 & 0 \\
\hline Ethyl lactate & $97-64-3$ & 1 & 1 \\
\hline Ethyl safranate & $35044-59-8$ & 1 & 1 \\
\hline Geranyl acetate & $105-87-3$ & 1 & 1 \\
\hline Heneicosane & $629-94-7$ & 1 & 1 \\
\hline Heptadecane & $629-78-7$ & 1 & 1 \\
\hline Heptadecyl acetate & $822-20-8$ & 1 & 0 \\
\hline Hexanal & $66-25-1$ & 1 & 1 \\
\hline Hexane* & $110-54-3$ & 1 & 1 \\
\hline Hexyl methyl ether & $4747-07-3$ & 1 & 1 \\
\hline Hexyl salicylate & $6259-76-3$ & 1 & 0 \\
\hline iso-Bornyl methacrylate & $7534-94-3$ & 1 & 1 \\
\hline Isomenthol acetate & $20777-45-1$ & 1 & 1 \\
\hline Isoprene (stabilised) & $78-79-5$ & 1 & 1 \\
\hline Isopropyl formate & $625-55-8$ & 1 & 1 \\
\hline Isovaleraldehyde & $590-86-3$ & 1 & 1 \\
\hline Isozonarol & $39707-55-6$ & 1 & 1 \\
\hline L-menthol & $2216-51-5$ & 1 & 0 \\
\hline Menthyl acetate & $89-48-5$ & 1 & 1 \\
\hline Methyl salicylate & $119-36-8$ & 1 & 1 \\
\hline Nonyl 2-methylpropanoate & $10522-34-6$ & 1 & 1 \\
\hline Nopyl acetate & $128-51-8$ & 1 & 1 \\
\hline
\end{tabular}




\begin{tabular}{|c|c|c|c|c|}
\hline o-Xylene* & $95-47-6$ & 1 & 0 & 1 \\
\hline Pentane & $109-66-0$ & 1 & 1 & 0 \\
\hline Phenethyl butyrate & $103-52-6$ & 1 & 1 & 0 \\
\hline Phenyl cyclobutanecarboxylate & $30466-31-0$ & 1 & 1 & 0 \\
\hline $\begin{array}{l}\text { Phenylacetaldehyde dimethyl } \\
\text { acetal }\end{array}$ & $101-48-4$ & 1 & 0 & 1 \\
\hline Phytane & $638-36-8$ & 1 & 1 & 0 \\
\hline Prenyl acetate & $1191-16-8$ & 1 & 1 & 0 \\
\hline Propanal* & $123-38-6$ & 1 & 1 & 0 \\
\hline Propylene glycol & $57-55-6$ & 1 & 1 & 0 \\
\hline Terpinyl acetate & $80-26-2$ & 1 & 1 & 0 \\
\hline Tetracarbonylnickel* & $13463-39-3$ & 1 & 1 & 0 \\
\hline Tetrahydrolinalool & $57706-88-4$ & 1 & 1 & 0 \\
\hline Thujopsene & $470-40-6$ & 1 & 1 & 0 \\
\hline Toluene* & $108-88-3$ & 1 & 1 & 0 \\
\hline trans-2-Octene & $13389-42-9$ & 1 & 1 & 0 \\
\hline trans-4-Octene & $14850-23-8$ & 1 & 1 & 0 \\
\hline Triacetin & $102-76-1$ & 1 & 1 & 0 \\
\hline Triethylene glycol & $112-27-6$ & 1 & 1 & 0 \\
\hline $\begin{array}{l}\alpha, \alpha \text {-Dimethylphenethyl } \\
\text { butyrate }\end{array}$ & $10094-34-5$ & 1 & 1 & 0 \\
\hline$\gamma$-Undecalactone & $104-67-6$ & 1 & 1 & 0 \\
\hline
\end{tabular}

*Compound classified as potentially hazardous 